\title{
Perioperative Care of a Pediatric Patient With PHACES Syndrome
}

\author{
Archana Ramesh ${ }^{\mathrm{a}}$, Richard Cartabuke ${ }^{\mathrm{a}}$, Joseph D. Tobias ${ }^{\mathrm{a}, \mathrm{b}, \mathrm{c}}$
}

\begin{abstract}
PHACE syndrome is a neurocutaneous syndrome that was originally described by Dr. Ilonia Frieden in 1996. The term PHACE is an acronym for the main components of the syndrome including: Posterior fossa malformations of the central nervous system (CNS); Hemangioma typically involving the skin of the head or neck; Arterial lesions usually involving the cerebral vasculature; Cardiac abnormalities, most commonly aortic coarctation; and Eye abnormalities. When accompanied by ventral developmental defects, it is termed PHACES syndrome, with the "S" representing sternal cleft or supraumbilical raphe. Patients may present for a variety of surgical procedures including the airway, CNS or the heart. Airway compromise secondary to hemangiomas and the potential for alterations in cerebral blood flow due to abnormalities of the cerebral arterial vasculature present major challenges for the anesthetic management of these patients. The perioperative implications of PHACES syndrome are discussed and previous reports of anesthetic care are reviewed.
\end{abstract}

Keywords: PHACES; Hemangiomia; Syndrome

\section{Introduction}

PHACE syndrome was originally described in 1996 by Dr. Ilonia Frieden [1]. Prior to this, Reese et al reported nine cases of hemangiomas associated with posterior fossa abnormalities including Dandy-Walker malformations [2]. In

\footnotetext{
Manuscript accepted for publication July 5, 2013

a'Department of Anesthesiology, Nationwide Children's Hospital and the Ohio State University, Columbus, Ohio, USA

${ }^{b}$ Department of Pediatrics, Nationwide Children's Hospital and the Ohio State University, Columbus, Ohio, USA

${ }^{\mathrm{c}}$ Corresponding author: Joseph D. Tobias, Department of

Anesthesiology \& Pain Medicine, Nationwide Children's Hospital,

700 Children's Drive, Columbus, Ohio 43205, USA.

Email: Joseph.Tobias@Nationwidechildrens.org

doi: http://dx.doi.org/10.4021/jmc1374w
}

several of the patients, associated arterial abnormalities were also noted. The term PHACE is an acronym for the main components of the syndrome including: Posterior fossa malformations of the central nervous system (CNS), particularly Dandy-Walker malformation and agenesis or hypoplasia of the cerebellum or cerebellar vermis; Hemangiomas, specifically segmental facial hemangiomas or airway hemangiomas; Arterial lesions usually involving the cerebral vasculature, which may present as agenesis, aneurysm, or occlusion of the carotid arteries and its branches; Cardiac abnormalities, most commonly aortic coarctation; and Eye abnormalities, specifically microphthalmos [3]. When accompanied by ventral developmental defects, it is termed PHACES syndrome, where the "S" represents sternal cleft or supraumbilical raphe. There is a female to male ratio of 8:1 suggesting that PHACES is an X-linked dominant disorder that causes in utero demise in the male fetus. The disorder is generally considered to be one of the neurocutaneous syndromes. Given the associated dermatologic and CNS involvement, it may be confused with Sturge-Weber syndrome (SWS) [4]. Distinguishing features of PHACE include hemangiomas as opposed to the capillary malformation (port wine stain) observed with SWS as well as cerebral arterial manifestations rather than the classical leptomeningeal involvement (angiomatosis or malformations) in SWS. Given the multi-system involvement of the disorder, patients may present for surgical procedures of the airway, CNS or the heart. The perioperative concerns of such patients are discussed and previous reports of anesthetic care are reviewed.

\section{Case Report}

Institutional Board Review is not required at Nationwide Children's Hospital (Columbus, Ohio) for the presentation of single case reports. The patient was a 2-year-old, $14.7 \mathrm{~kg}$ child with PHACE syndrome who presented with subglottic hemangiomas, chronic otitis media, sleep-disordered breathing, and tonsillar hypertrophy. He was originally diagnosed with PHACE syndrome in infancy, manifestations of which included cutaneous hemangioma on the cheek, lower lip, mucosal surfaces, and left mandibular angle; a subglottic 
hemangioma, intracranial arterial tortuosity, and a sternal cleft. He also had a history of episodes of stridor which had not required therapy. The patient was the product of a fullterm gestation with spontaneous vaginal delivery and a birth weight of $3.5 \mathrm{~kg}$. There were no prenatal problems. Congenital heart disease (CHD) included a patent foramen ovale and a patent ductus arteriosus, both of which had resolved spontaneously by 3 months of age. Past surgical procedures included direct laryngoscopy and bronchoscopy with triamcinolone injection into the subglottic hemangioma, bilateral ear tube insertion, and adenoidectomy at the age of 15 months. Magnetic resonance imaging performed at 1.5 years revealed arterial tortuosity affecting the posterior distribution of the cerebral vasculature, particularly the basilar artery and the superior cerebellar arteries with smaller circumferential vessels involved as well. The tortuosity appeared most prominent at the interpeduncular cistern with some extension to the right ambient cistern. Magnetic resonance angiography 6 months later demonstrated the abnormal vessels filling the cisterns, as described above, without significant change from the previous examination. Additionally, there was abrupt narrowing of the terminal segments of the internal carotid and basilar arteries. Apart from stuttering, he was developmentally appropriate for age with a normal neurological exam.

The child was admitted on the morning of the surgery for bilateral examination under anesthesia of the external auditory canals and tympanic membranes, direct laryngoscopy, rigid bronchoscopy, and tonsillectomy. He was held nil per os (NPO) for $6 \mathrm{~h}$. His preoperative vital signs revealed an oxygen saturation of $97 \%$, blood pressure of $112 / 63 \mathrm{mmHg}$, and a heart rate of 112 beats/min. He was transported to the operating room and routine monitors were applied according to American Society of Anesthesiologists' guidelines. Anesthesia was induced with sevoflurane in oxygen. Following anesthetic induction, a 20 gauge peripheral intravenous cannula was placed and propofol $(15 \mathrm{mg})$ was administered. Following the topical application of lidocaine $(50 \mathrm{mg})$ to the trachea, direct laryngoscopy was performed while maintaining spontaneous ventilation with the insufflation of sevoflurane via the laryngoscope and subsequently the rigid bronchoscope. No significant abnormalities were observed. The trachea was intubated with a $3.5 \mathrm{~mm}$ cuffed endotracheal tube (ETT) following completion of the bronchscopy. With the cuff deflated, an airleak around the ETT was noted at 25 $\mathrm{cmH}_{2} \mathrm{O}$. Breath sounds were equal and bilateral with positive end-tidal carbon dioxide recorded by capnography. Anesthesia was maintained with sevoflurane (expired concentration $2-4 \%$ ) in a combination of air and oxygen to maintain mean arterial pressure (MAP) within 25\% of preoperative values. Fentanyl $(25 \mu \mathrm{g})$ and acetaminophen $(250 \mathrm{mg})$ were administered for postoperative analgesia. Additional medications included dexamethasone $(0.25 \mathrm{mg} / \mathrm{kg})$ and ondansetron $(0.15$ $\mathrm{mg} / \mathrm{kg}$ ). Estimated blood loss was $25 \mathrm{~mL}$. Total intraopera- tive fluids totaled $300 \mathrm{~mL}$. Following the surgical procedure, the patient's trachea was extubated and he was transferred to the post-anesthesia care unit. He was weaned to room air and transferred to the inpatient ward for postoperative monitoring with continuous pulse oximetry. Postoperative analgesia was provided by a combination of oral acetaminophen and hydrocodone elixir. His postoperative course was unremarkable and he was discharged home the following day.

\section{Discussion}

There are several perioperative concerns to consider when planning anesthetic care for patients with PHACE syndrome. As with any anesthetic care, appropriate preoperative preparation begins with a thorough history and physical examination. Of primary concern to anesthesia providers is the associated presence of airway hemangiomas which may complicate airway management. Extracutaneous hemangiomas occur in approximately $25 \%$ of children with PHACE syndrome with subglottic hemangiomas being the most common. Durr et al retrospectively reviewed their experience with airway hemangiomas in 23 patients with PHACE syndrome [5]. Twelve of the 23 patients (52\%) had documented airway hemangiomas. Six of the patients underwent airway examination in the operating room at their institution. Five $(83 \%)$ of the patients had subglottic hemangiomas while three $(50 \%)$ had additional hemangiomas within the airway located on the epiglottis, vocal folds, posterior pharyngeal wall, and tracheal wall. Treatment included propranolol, corticosteroids, and vincristine. Although one patient required tracheostomy for airway obstruction, all were symptom free at follow-up which ranged from 13 to 76 months. The authors noted the frequent occurrence of potentially life-threatening airway hemangiomas in patients with PHACE syndrome and concluded patients with a history of respiratory symptoms, such as unexplained tachypnea, stridor, or hoarseness should be referred for airway evaluation. Depending on the patient's status, this may include awake nasal endoscopy to evaluate the upper airway or more commonly, direct laryngoscopy, and bronchoscopy in the operating room under general anesthesia [6].

The appropriate equipment for dealing with the difficult airway should be readily available prior to anesthetic induction, as recommended by Engelehart [7]. General anesthesia can be induced by the inhalation of sevoflurane in $100 \%$ oxygen with the maintenance of spontaneous ventilation. Following anesthetic induction, general anesthesia is maintained with spontaneous ventilation via insufflation of sevoflurane in $100 \%$ oxygen through the distal port of the operating laryngoscope and through the rigid bronchoscope. Neuromuscular blocking agents are generally avoided. The administration of an anticholinergic agent may be helpful to prevent reflex bradycardia associated with airway stimula- 
tion as well as reducing airway secretions to improve visualization [8]. However, this should be weighed against the need to prevent changes in MAP or heart rate in patients with significant cerebrovascular disease. Topical lidocaine (3 - 5 $\mathrm{mg} / \mathrm{kg}$ of a $2-4 \%$ solution) can be applied to the airway to supplement anesthesia prior to airway instrumentation. The preoperative medical therapy employed in the treatment of airway hemangiomas may impact perioperative care. $\mathrm{Pa}$ tients on prolonged corticosteroid therapy may require the administration of a stress dose of hydrocortisone, depending on the duration of treatment and the extent of the surgical procedure. Propranolol is a mainstay of treatment for infantile hemagiomas with a reported response rate of $98 \%$ [9]. Propranolol may be held the day of surgery given concerns regarding its hemodynamic effects with potentiation of intraoperative bradycardia or hypoglycemia. However, prolonged withdrawal of therapy may lead to rebound tachycardia and hypertension, which may be deleterious in the patient with significant cerebrovascular disease. Other less common therapy for hemangiomas such as vincristine may result in autonomic or motor neuropathy. Although this rarely impacts anesthetic care, vincristine has been reported to cause isolated vocal cord paresis [10].

Another major concern of children with PHACE syndrome, as noted in our patient, is the presence of arterial abnormalities with cerebral and cervical arterial malformations being the most common [11]. The arteriopathy most commonly involves the internal carotid artery and its embryonic branches, followed by the middle cerebral artery. In addition to anomalous origin and abnormal course of these vessels, hypoplasia, tortuosity, stenosis, and occlusion have been reported. These anomalies are generally located on the ipsilateral side of the cutaneous hemangiomas. Persistent embryonic arteries especially of the trigeminal artery and Moyamoya phenomena have also been reported [12]. In a review of 39 case series with a total of 115 children with PHACE syndrome by Heyer et al, 89 of the 115 patients $(77.4 \%)$ had cerebrovascular anomalies. Although the exact cause for the arterial anomalies is not clear, it has been proposed that the cerebral vascular development in the early embryonic period ( $\leq 5$ weeks) is affected [12]. Burrows et al, in a retrospective study of eight children with cervicofacial hemangiomas and intracranial arterial anomalies, proposed that the arterial malformations may be congenital or develop later in life [13]. This involvement may also be progressive [14]. Although neurological sequelae are rare, the clinical impact may be significant when acute ischemic stroke occurs. This may present as new-onset seizures or acute hemiparesis. The incidence of such problems generally coincides with the time of the most rapid growth of the hemangioms (6 - 18 months of age). Since abnormal cerebral vasculature is a major risk factor for arterial ischemic stroke, it has been suggested that children with PHACE syndrome should undergo neuroimaging to view the arterial architecture of the brain $[14,15]$.
There is no evidence-based support for the medical treatment of PHACES patients at risk for acute ischemic stroke [16]. However, prophylactic treatment regimens may include the use of anti-platelet medications such as acetylsalicylic acid (aspirin). Consultation with surgery, cardiology, and neurology may be required to determine a perioperative plan regarding the continuation of such therapy.

The predisposition of these patients to acute ischemic stroke and seizures mandates that appropriate attention be focused perioperatively on factors that affect cerebral blood flow $(\mathrm{CBF})$ and oxygen delivery. The anesthetic regimen should maintain the appropriate depth of anesthesia while minimizing hemodynamic effects that alter cerebral perfusion pressure and CBF. Hemodynamic depression may occur throughout the anesthetic care, but is most likely to occur during anesthetic induction. Perioperative hypotension should be treated promptly with a direct acting $\alpha$-adrenergic agonist such as phenylephrine $(1-2 \mu \mathrm{g} / \mathrm{kg})$. Potential adverse effects related to intraoperative hypocarbia, such as decreases in $\mathrm{CBF}$, suggest that intraoperative hyperventilation should be avoided [17-19]. These changes may have greater clinical significance in patients with decreased CBF related to ischemic disease processes. Javault et al suggested neuromonitoring with the use of near infrared spectroscopy, as well as the bispectral index, when there are concerns regarding $\mathrm{CBF}$ and the potential for cerebral ischemia [20]. In specific clinical scenarios, transcranial ultrasound and Doppler may also be used to complement near-infrared spectroscopy in assessing cerebral perfusion.

Although our patient did not have a history of seizures requiring anticonvulsant therapy, seizures may accompany the arteriopathy of PHACE syndrome. Preoperative management to limit the incidence of perioperative seizures includes optimizing anticonvulsant therapy and documentation of therapeutic anticonvulsant levels prior to surgery. Routine anticonvulsant medications should be administered the morning of the procedure despite concerns of the patient's NPO status. Maintenance doses of anticonvulsant agents should be administered intraoperatively and postoperatively. As enteral administration may not be feasible, alternative routes of delivery (intravenous or transrectal) should be considered. Consultation with the neurology or pharmacology service is suggested when questions arise concerning dosing conversion from enteral to intravenous administration or intraoperative redosing.

The child with PHACE syndrome also has the potential for associated cardiovascular anomalies. Our patient had a history of a patent foramen ovale and patent ductus arteriosus, which had closed spontaneously by 3 months of age. However, clinically significant cardiovascular lesions are not uncommon in these patients. Analysis of data from 50 children with PHACE syndrome revealed that $37 \%$ of these had cardiovascular anomalies, with aortic coarctation and aberrant subclavian artery being the most common [3]. The 
coarctation of the aorta that occurs in children with PHACE syndrome is atypical. Unlike the classical type, there is involvement of the aorta proximal to or at the level of the origin of great vessels. Hence, the classic blood pressure gradient observed between the upper and lower extremities may not be present. The aortic segment involved in these children may be much longer compared to relatively discrete involvement of the classical type. The other cardiac abnormalities reported include aortic arch abnormalities, tetralogy of Fallot, septal defects, and stenosis of the semilunar valves [21].

The exact perioperative care of patients varies, depending on the type of CHD that is present. Screening for the presence of a coarctation during the preoperative evaluation includes auscultation of the heart as well as blood pressure (BP) and pulse checks in all four extremities. Echocardiography and consultation with a cardiologist should be considered, based on the nature of the CHD. Anesthetic goals focus on reducing the cardiovascular stress proximal to the obstruction by maintaining a normal heart rate and left ventricular filling pressure, ensuring adequate perfusion of the tissues, and control of the systemic vascular resistance [22, 23]. BP monitoring at two points (proximally and distally to the obstruction) should be considered to ensure adequate BP control pre-coarctation and maintenance of an adequate $\mathrm{BP}$ distal to the obstruction to ensure adequate perfusion pressure.

Ocular manifestations have been reported in $16 \%$ of PHACE patients [3]. These include microphthalmia, optic nerve hypoplasia, cataracts, and increased retinal vascularity [1]. Since endocrine anomalies, commonly those of the thyroid and pituitary, have been reported in children with PHACE syndrome, it has been suggested that the "E" in "PHACE" should reflect endocrinopathy along with eye manifestations. The hypothyroidism found in PHACE syndrome may be due dysfunction at several levels of the hypothalamus-pituitary-thyroid axis [24, 25]. Additional reports document the association of PHACES with a lingual thyroid gland [25]. Additional causes for thyroid dysfunction include the interferon therapy used in the treatment of hemangioma as well as the enzyme, 3-iodothyronine deiodinase, secreted by the hemangioma [26-28]. A high index of suspicion in these children with endocrinology consultation may be helpful in the timely diagnosis of the thyroid dysfunction in patients with PHACE syndrome. In patients with hypothalamic and pituitary dysfunction resulting in hypothyroidism, adrenocortical function should also be evaluated given its impact on perioperative outcome [29-31].

In summary, PHACE syndrome is a recently described neurocutaneous syndrome. Given the end-organ involvement, various surgical procedures may be required in these patients. Specific perioperative concerns include potential difficulties with airway management related to airway hemangiomas as well as an arteriopathy which predominantly affects the carotid vasculature and may impact on CBF re- sulting in ischemia, and associated CHD. The assessment of end-organ impairment by the primary disease process during the preoperative evaluation will allow for the effective perioperative care of these patients.

\section{References}

1. Frieden IJ, Reese V, Cohen D. PHACE syndrome. The association of posterior fossa brain malformations, hemangiomas, arterial anomalies, coarctation of the aorta and cardiac defects, and eye abnormalities. Arch Dermatol. 1996;132(3):307-311.

2. Reese V, Frieden IJ, Paller AS, Esterly NB, Ferriero D, Levy ML, Lucky AW, et al. Association of facial hemangiomas with Dandy-Walker and other posterior fossa malformations. J Pediatr. 1993;122(3):379-384.

3. Metry DW, Garzon MC, Drolet BA, Frommelt P, Haggstrom A, Hall J, Hess CP, et al. PHACE syndrome: current knowledge, future directions. Pediatr Dermatol. 2009;26(4):381-398.

4. Billson VR, Gillam GL. An unusual case of Sturge-Weber syndrome. Pathology. 1984;16(4):462-465.

5. Durr ML, Meyer AK, Huoh KC, Frieden IJ, Rosbe KW. Airway hemangiomas in PHACE syndrome. Laryngoscope. 2012;122(10):2323-2329.

6. English J, Norris W, Bedforth N. Anaesthesia for airway surgery. Contin Educ Anaesth Crit Care Pain. 2006;6:2831.

7. Engelhardt T, Weiss M. A child with a difficult airway: what do I do next? Curr Opin Anaesthesiol. 2012;25(3):326-332.

8. Gronnebech H, Johansson G, Smedebol M, Valentin N. Glycopyrrolate vs. atropine during anaesthesia for laryngoscopy and bronchoscopy. Acta Anaesthesiol Scand. 1993;37(5):454-457.

9. Chen TS, Eichenfield LF, Friedlander SF. Infantile hemangiomas: an update on pathogenesis and therapy. Pediatrics. 2013;131(1):99-108.

10. Tobias JD, Bozeman PM. Vincristine-induced recurrent laryngeal nerve paralysis in children. Intensive Care Med. 1991;17(5):304-305.

11. Hess CP, Fullerton HJ, Metry DW, Drolet BA, Siegel DH, Auguste KI, Gupta N, et al. Cervical and intracranial arterial anomalies in 70 patients with PHACE syndrome. AJNR Am J Neuroradiol. 2010;31(10):19801986.

12. Heyer GL, Dowling MM, Licht DJ, Tay SK, Morel K, Garzon MC, Meyers P. The cerebral vasculopathy of PHACES syndrome. Stroke. 2008;39(2):308-316.

13. Burrows PE, Robertson RL, Mulliken JB, Beardsley DS, Chaloupka JC, Ezekowitz RA, Scott RM. Cerebral vasculopathy and neurologic sequelae in infants with cervicofacial hemangioma: report of eight patients. Ra- 
diology. 1998;207(3):601-607.

14. Siegel DH, Tefft KA, Kelly T, Johnson C, Metry D, Burrows P, Pope E, et al. Stroke in children with posterior fossa brain malformations, hemangiomas, arterial anomalies, coarctation of the aorta and cardiac defects, and eye abnormalities (PHACE) syndrome: a systematic review of the literature. Stroke. 2012;43(6):1672-1674.

15. Luo CB, Lasjaunias P, Teng MM, Chang FC, Lirng JF, Chang CY. Cervico-cerebrovascular anomalies in children with PHACE syndrome. J Formos Med Assoc. 2003;102(6):379-386.

16. Hartemink DA, Chiu YE, Drolet BA, Kerschner JE. PHACES syndrome: a review. Int J Pediatr Otorhinolaryngol. 2009;73(2):181-187.

17. Rozmiarek A, Taghon T, Tobias JD. Inadvertent hyperventilation during intraoperative anesthetic care in the pediatric population. ICU Director. 2012;4:172-175.

18. Kameyama M, Shirane R, Tsurumi Y, Takahashi A, Fujiwara S, Suzuki J, Ito M, et al. Evaluation of cerebral blood flow and metabolism in childhood moyamoya disease: an investigation into "re-build-up" on EEG by positron CT. Childs Nerv Syst. 1986;2(3):130-133.

19. Kurehara K, Ohnishi H, Touho H, Furuya H, Okuda T. Cortical blood flow response to hypercapnia during anaesthesia in Moyamoya disease. Can J Anaesth. 1993;40(8):709-713.

20. Javault A, Metton O, Raisky O, Bompard D, Hachemi $\mathrm{M}$, Gamondes D, Ninet J, et al. Anesthesia management in a child with PHACE syndrome and agenesis of bilateral internal carotid arteries. Paediatr Anaesth. 2007;17(10):989-993.

21. Bijulal S, Sivasankaran S, Krishnamoorthy KM, Titus T, Tharakan JA, Krishnamanohar SR. Unusual coarctationthe PHACE syndrome: report of three cases. Congenit Heart Dis. 2008;3(3):205-208.

22. Zwiers WJ, Blodgett TM, Vallejo MC, Finegold H. Successful vaginal delivery for a parturient with complete aortic coarctation. J Clin Anesth. 2006;18(4):300-303.
23. Sinha R, Garg R. Anesthetic management for laparoscopy surgery in a patient with residual coarctation of aorta and mild aortic stenosis. J Anaesthesiol Clin Pharmacol. 2011;27(3):412-413.

24. Poindexter G, Metry DW, Barkovich AJ, Frieden IJ. PHACE syndrome with intracerebral hemangiomas, heterotopia, and endocrine dysfunction. Pediatr Neurol. 2007;36(6):402-406.

25. Majumdar I, Mastrandrea LD. Lingual thyroid as a cause of primary hypothyroidism: congenital hypothyroidism in the neonatal period and beyond. Clin Pediatr (Phila). 2010;49(9):885-888.

26. Abdel-Kader ME, Azmy MK, Hassan MA, Ahmad YK, El-Dahshan T, Houssein MA. Thyroid function in patients with chronic hepatitis $\mathrm{C}$ virus infection under interferon therapy. J Egypt Soc Parasitol. 2012;42(3):761768.

27. Marcellin P, Pouteau M, Renard P, Grynblat JM, Colas Linhart N, Bardet P, Bok B, et al. Sustained hypothyroidism induced by recombinant alpha interferon in patients with chronic hepatitis C. Gut. 1992;33(6):855-856.

28. Huang SA, Tu HM, Harney JW, Venihaki M, Butte AJ, Kozakewich HP, Fishman SJ, et al. Severe hypothyroidism caused by type 3 iodothyronine deiodinase in infantile hemangiomas. N Engl J Med. 2000;343(3):185-189.

29. Stathatos N, Wartofsky L. Perioperative management of patients with hypothyroidism. Endocrinol Metab Clin North Am. 2003;32(2):503-518.

30. Zada G, Woodmansee WW, Iuliano S, Laws ER. Perioperative management of patients undergoing transsphenoidal pituitary surgery. Asian J Neurosurg. 2010;5(1):16.

31. Esposito F, Dusick JR, Cohan P, Moftakhar P, McArthur D, Wang C, Swerdloff RS, et al. Clinical review: Early morning cortisol levels as a predictor of remission after transsphenoidal surgery for Cushing's disease. J Clin Endocrinol Metab. 2006;91(1):7-13. 